\section{PTH-4 CAPSULE ENDOSCOPY; DISTRICT GENERAL HOSPITAL EXPERIENCE IN EAST KENT TRUST}

1,25oad Elkady*, 'Laura Crisan, ${ }^{1}$ Carlo Nunes. 'East Kent Hospitals University Foundation NHS Trust, Canterbury, Kent, UK; ${ }^{2}$ University of Alexandria, Alexandria, Egypt

\subsection{6/gutjnl-2021-BSG.283}

Introduction Capsule endoscopy (CE) has revolutionized smallbowel (SB) examination by providing a reliable and non-invasive method for complete visualization and assessment of SB mucosal surface.

Methods A retrospective study to assess CE service in East Kent Trust was performed over the period between June 2016 till January 2019. All CE reports, previous endoscopies and magnetic resonance enterography (MRE) prior to CE were reviewed

Results 328 patients were referred for CE in the study period. Most common indication was iron deficiency anaemia (IDA) in $50 \%$, followed by suspected Crohn's Disease (CD) in $27 \%$. Bidirectional endoscopy was done in 215 (65\%) cases and MRE was done in 102 cases (31\%). SB transit time ranged between 25 minutes to $8 \mathrm{~h} 5$ minutes. Caecal visualization was achieved in $90 \%$ of cases, while only two patients $(0.6 \%)$ had retained $\mathrm{CE}$ in the stomach. The study was normal in 181 cases (55\%), while SB pathology was detected in 147 cases (45\%). Most common findings were aphthous ulcers in 34 cases $(10 \%)$ and angioectasia in 32 cases(10\%). The diagnostic yield was higher for obscure gastrointestinal bleeding (OGIB) indication than for IDA indication (67\% versus 47\%). The frequency of P1 and P2 lesions was higher in OGIB [P1 5 cases $(21 \%)$, P2 9 cases $(37 \%)]$ than in IDA indication [P1 22 cases (13\%), P2 32cases (20\%)]. The diagnostic yield for suspected SB CD indication was 40\%, while it was 50\% for assessment of $\mathrm{CD}$ indication. Double balloon enteroscopy (DBE) was recommended in 38 cases (12\%). Histopathological

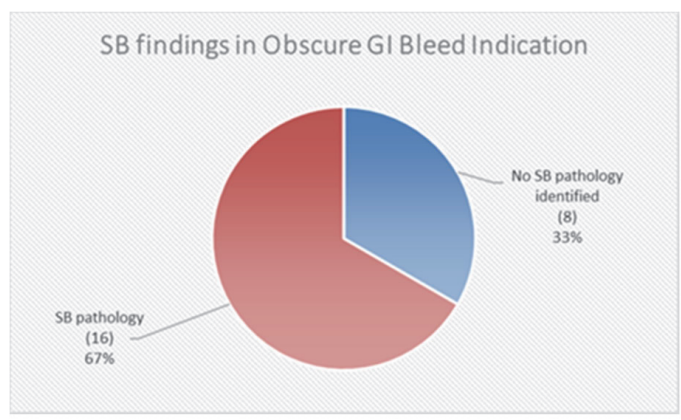

a

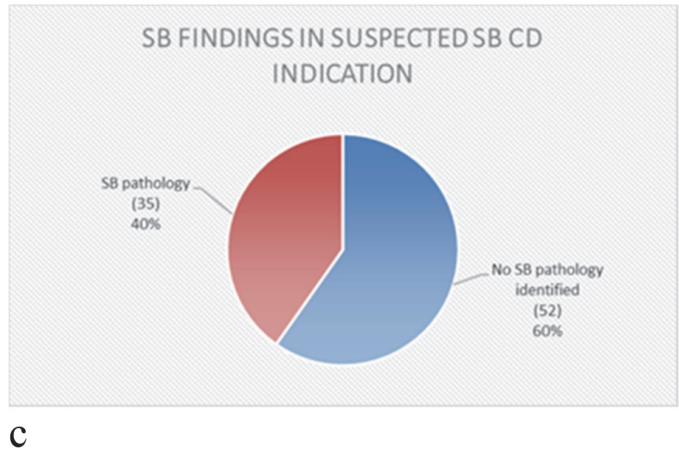

diagnosis was achieved in 35\% of cases by either enteroscopy or colonoscopy. CE findings were confirmed by SB biopsies (bx) in $18 \%$ of cases in comparison to $11 \%$ in MRE. CE correlated with MRE showing similar findings in 14\% of cases. Sensitivity and specificity of CE for detection of CD were $76.92 \%$ CI $(56.35 \%$ to $91.03 \%)$ and $78.43 \%$ CI $(64.68 \%$ to $88.71 \%$ ) respectively with SB bx taken as gold standard.

Conclusions In our cohort CE correlated more with SB bx than MRE. Considering the key performance indicators (KPI) proposed by European Society of Gastrointestinal Endoscopy by Spada et al (2019) as reference, our service achieved target standards in most KPI [indications 97\% (standard $\geq 95 \%$ ), caecal visualization $90 \%$ (standard $\geq 80 \%$ ), and capsule retention $0.6 \%$ (standard $\leq 2 \%)]$. Only 38 patients $(12 \%)$ were referred for DBE at tertiary centre, which is less than the standard. Lesion detection standard $(\geq 50 \%)$ was achieved in OGIB (67\%) and assessment of CD (50\%) indications but was

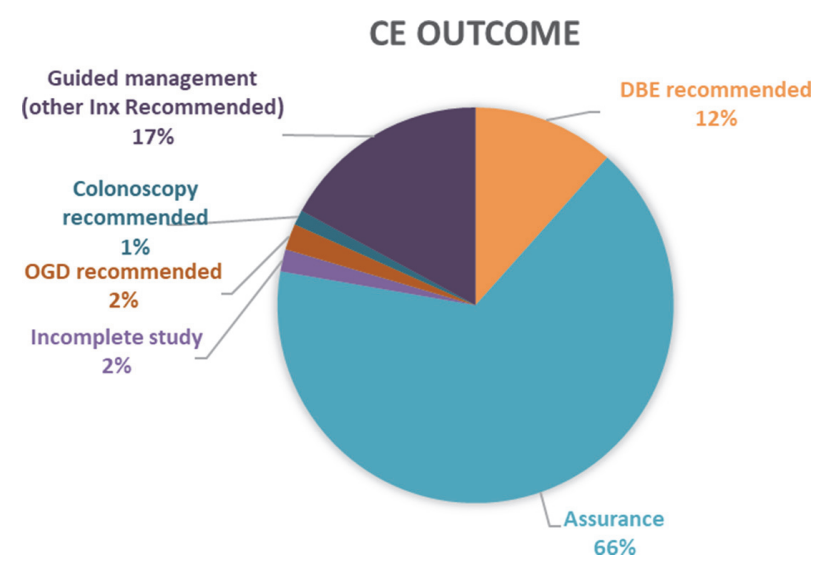

Abstract PTH-4 Figure 2 CE outcome
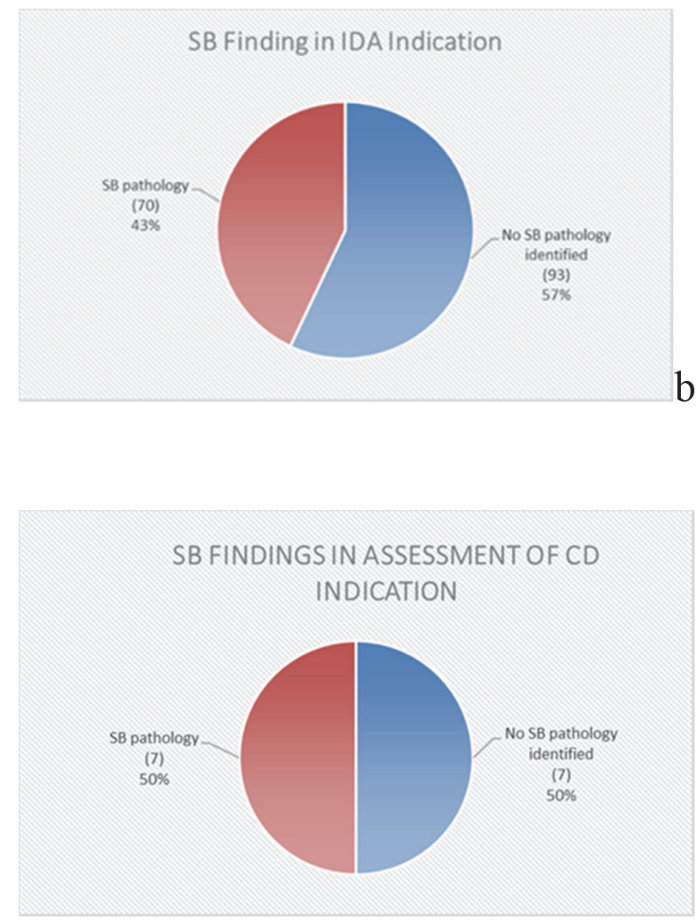

d

Abstract PTH-4 Figure 1 CE yield according to indication 
slightly lower than target in IDA (43\%) and suspected CD $(40 \%)$ indications, reflecting that better selection of cases can increase CE diagnostic yield. A standardized approach to audit $\mathrm{CE}$ is necessary and should follow KPI as a standard for comparison.

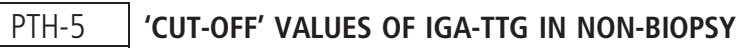 DIAGNOSTIC PATHWAYS FOR COELIAC DISEASE NEED TO BE ASSAY-SPECIFIC}

Richard Vaughan*, Francesca Melindo, Benjamin Corney, Hannah Williams, Alice Bone, James Berrill. Royal Glamorgan Hospital, Llantrisant, UK

\subsection{6/gutjpl-2021-BSG.284}

Introduction The British Society of Gastroenterology (BSG) interim guidelines (June 2020) on making a non-biopsy diagnosis of coeliac disease included an IgA anti-tissue transglutaminase (TTG) level $\geq 10 \times$ upper limit of normal (ULN) in the pathway. However this was only recommended for an ELISA-based assay. It advised that local TTG assay reliability needs correlating to pathology as part of any local audit.

Since June 2019, our health board switched from an ELISA based assay to a chemiluminescence based assay for TTG measurement. This study aims to compare the two distinct assays, and propose an appropriate 'cut-off' level for the chemiluminescence assay.

Method In this retrospective, observational study across two sites, all elevated TTG results in adult patients from January 2018 to December 2019 were identified from biochemistry records. Patients already known to have coeliac disease were excluded from analysis. In patients with multiple elevated TTG results only the earliest result was included. Data was collected on patient demographics, waiting times, and duodenal biopsy results. Prior to June 2019 an ELISA based assay was used with TTG results $<10 \mathrm{U} / \mathrm{ml}$ considered negative. Since June 2019 a chemiluminescence assay (Bioflash) was employed, with TTG results $<20 \mathrm{U} / \mathrm{ml}$ reported as negative.

Results In total, 192 elevated TTG results were included in the analysis. One hundred and nine patients had duodenal biopsies performed, 73 patients were not referred or declined investigation, and 10 patients were still waiting for duodenal biopsy at the time of analysis in May 2021. The median waiting time for these 10 patients still awaiting biopsy was 456 days.

Using the ELISA based assay, $96 \%$ patients $(n=27)$ with TTG $>10 \times$ ULN had positive duodenal biopsies, whereas positive biopsies were only present in $56 \%$ patients $(n=9)$ with TTG between $5-10$ x ULN.

Using the Chemiluminescence assay, 90\% patients $(n=21)$ with TTG $>10 \times$ ULN had positive duodenal biopsies, however positive biopsies were also present in $89 \%$ patients $(n=9)$ with TTG between 5 - 10 x ULN.

Discussion These results support the BSG guidance for nonbiopsy diagnosis of coeliac disease using a pathway that includes TTG $>10 \mathrm{x}$ ULN measured by an ELISA assay. However for the chemiluminescence assay (Bioflash) it appears that a TTG level $\geq 5 \mathrm{x}$ ULN may be a more appropriate 'cut-off' level to use in the pathway. Further work is required to confirm this as the numbers in this study are small. This study also shows the long time periods that patients in some centres are waiting for their duodenal biopsies, likely exacerbated by the COVID pandemic, and thus the importance of establishing a non-biopsy protocol where appropriate.

\section{PTH-6 UTILITY OF A HOME HYDROGEN BREATH TESTING PATHWAY FOR DIAGNOSIS OF SMALL INTESTINAL BACTERIAL OVERGROWTH}

Jabed Ahmed*, Pritpal Padam, Aruchuna Ruban. Imperial Healthcare NHS Trust, London, UK

\subsection{6/gutjnl-2021-BSG.285}

Introduction Small Intestinal bacterial overgrowth (SIBO) can lead to persistent diarrhoea, bloating and abdominal pain in patients. Hydrogen Breath testing (HBT) is the first line investigation but since the COVID-19 pandemic its utility has been limited as it is considered an aerosol generating procedure. We piloted a new testing pathway at a London teaching hospital in which HBT kits were sent out for patients to perform the test at home and sent back for analysis.

Aims \& Methods Patients on our HBT waiting list between April 2019 to November 2020 were contacted and assessed for symptomology, validity for home testing and consent for participation. 39 tests were mailed out to patients and data was collected on the response rate, number of positive results, alongside a patient satisfaction survey.

Results A total of 108 patients (M 42: F 66) were identified to be on the waiting list for a hydrogen breath test. 8 patients were removed due to symptom improvement, being discharged from clinic or due to cancellation of request. All 100 patients were contacted with 91 responding. 88 (96.7\%) agreed to undergo the home testing for SIBO. 86.4\% (76/88) reported to still be symptomatic with $13.6 \%(12 / 88)$ reporting no longer having symptoms.

39 home testing kits were sent and 14 (36\%) kits were returned over a 2 month period.

$10(26 \%)$ were hydrogen breath test positive. (Figure 1).

The average score given for ease of use for the home kit was $3.7 / 5.10 / 14(71 \%)$ of the patients accessed the video link and $4.3 / 5$ said they would recommend this testing method to others.

A follow up call was undertaken to ascertain the poor response rate from patients and common feedback included difficulty in understanding instructions, restrictive diet required prior to testing and time constraints.

Conclusions The initial results from our pilot study show promise and suggest that home testing is a realistic, viable and cost-effective first line option for the management of SIBO in the post covid era.

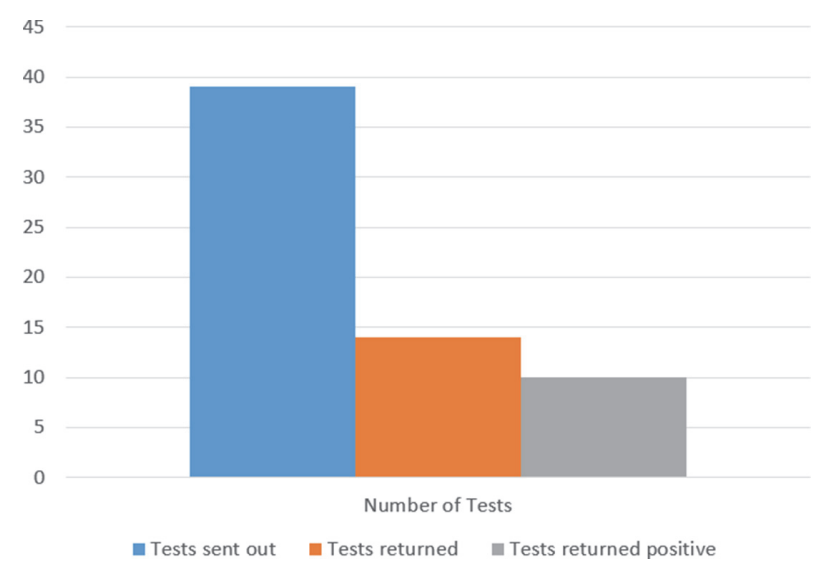

Abstract PTH-6 Frigure 1 Home breath tests 